\title{
Emprendimiento de Componentes Constructivos Paramétricos
}

\section{Entrepreneurship of Building Parametric Components}

\author{
Omar Rivera \\ U. del Bío-Bío, Chile \\ arma_rives@ymail.com \\ Alexis Salinas \\ U. del Bío-Bío, Chile \\ asalinas@alumnos.ubiobio.cl \\ Paula Ulloa \\ Galaxia Arquitectos \\ paulaignacia@gmail.com \\ Oscar Otárola \\ PixelArq \\ otarola.mardones@gmail.com
}

\author{
Braulio Gatica \\ U. del Bío-Bío, Chile \\ brauliogatica@hotmail.com \\ William Fuentes \\ U. del Bío-Bío, Chile \\ wfuentesl@gmail.com \\ Rodrigo García Alvarado \\ U. del Bío-Bío, Chile \\ rgarcia@ubiobio.cl \\ Mauro Chiarella \\ U. Nacional del Litoral, Argentina \\ mchiarella@hotmail.com
}

\begin{abstract}
New technologies of parametric design and digital fabrication encourages development of building components, involving a new approach to architectural work and promote professional endeavors. This paper presents several experiences of recent graduates of architecture in U. Bio-Bio, Chile, which demonstrate these capabilities. The development of a roof module, a structural grid, a flexible envelope, cutting partitions and folded units for wind protection are examples of mass-customization, with implementation of new processes of design, construction and divulgation into the building industry.
\end{abstract}

Keywords: Parametric Design; Digital Fabrication; Building Construction; Entrepreneurship; Innovative Architecture.

\section{Introducción}

Las nuevas tecnologías de modelación paramétrica y fabricación digital permiten ejecutar elementos sofisticados para edificios singulares (como se expone en Stacey, 2004 y Woodbury, 2010), pero también componentes variables y económicos para la construcción general (como lo demuestra Kieran y Timberlake, 2004; Gramazio y Kohler, 2008, García y Turkienicz, 2010), con el fin de aprovechar las capacidades de variación, optimización y masificación industrial. Logrando elementos innovadores con atributos de desempeño más eficaces, y eventualmente menores costos, a partir de experiencias educacionales (García et al, 2009), que promueven el desarrollo colectivo (Davis et al, 2011).

Este trabajo expone distintas experiencias en componentes paramétricos y su desarrollo general a través de emprendimientos profesionales. Exponiendo un proceso de diseño basado en la producción múltiple de los componentes, concebidos como un sistema variable y masificable.

Lo que implica una conceptualización de elementos aplicables en distintas situaciones, con materiales manufacturados de bajo costo, que otorguen mejores prestaciones que los productos convencionales, integrándose cabalmente en las edificaciones y sistemas constructivos prevalecientes. Lo que debe pasar por una formulación preliminar, una revisión tecnológica, modelos de pruebas y propuestas de comercialización. Planteando alternativas de desarrollo, capacidades de producción y materiales específicos, campos de aplicación y un modelo de negocios. Estableciendo un sistema de diseño paramétrico que recoja estas condicionantes y permita elaborar los componentes en distintos equipos $y$ materiales.

\section{Componentes Desarrollados}

En los últimos tres años varios egresados de la Esc. de Arquitectura de la U. Bío-Bío han desarrollado nuevos componentes constructivos basados en diseño paramétrico y fabricación digital, como emprendimientos profesionales.

\section{Tulipas}

Módulo de techumbre en estructura de madera y cubierta textil, que puede adaptarse a distintas configuraciones ambientales (Fig.1). Con un soporte de bajo costo industrializable, acoplable entre varias unidades y factible de instalar de manera permanente o temporal. Iniciado como una investigación universitaria multidisciplinaria, se han ejecutado diversos modelos y prototipos a escala real con apoyo de empresas del área. Actualmente se desarrolla como seminario de titulación por Omar Rivera y se postula a fondos públicos. 


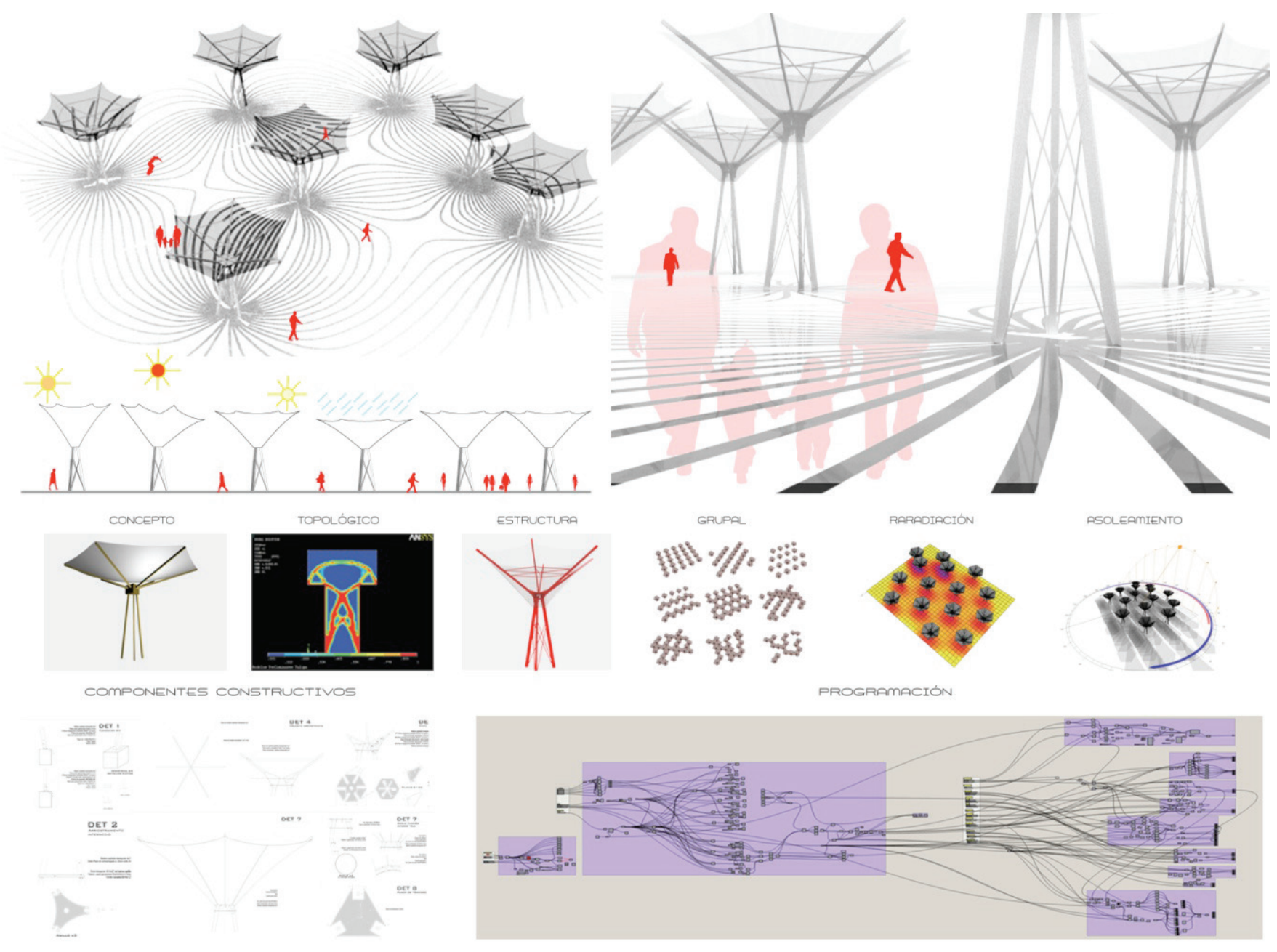

Figura 1: Desarrollo de Tulipas

\section{PixelArq}

Pixelarq es un proyecto de emprendimiento basado en el diseño paramétrico y fabricación digital (Fig.2), que surge de la experiencia en investigación y desarrollo del sistema "muro pixel", como seminario en 2009 y proyecto de título en 2010-2011, del arquitecto Oscar Otárola Mardones, dirigido por el Dr. Rodrigo García Alvarado. Actualmente, el proyecto ha recibido financiamiento de dos instituciones gubernamentales de fomento a la investigación y el emprendimiento tecnológico, lo que ha permitido desarrollar nuevos prototipos de mobiliario, paneles decorativos y elementos de protección solar para fachadas, aumentando las horas de experiencia en el manejo de software y estrategias de diseño paramétrico vinculadas a fabricación digital en fresadora cnc.

\section{Malla (grid-shell)}

En la necesidad de buscar sistemas constructivos que se adapten a las formalidades no tradicionales que permiten los procesos de
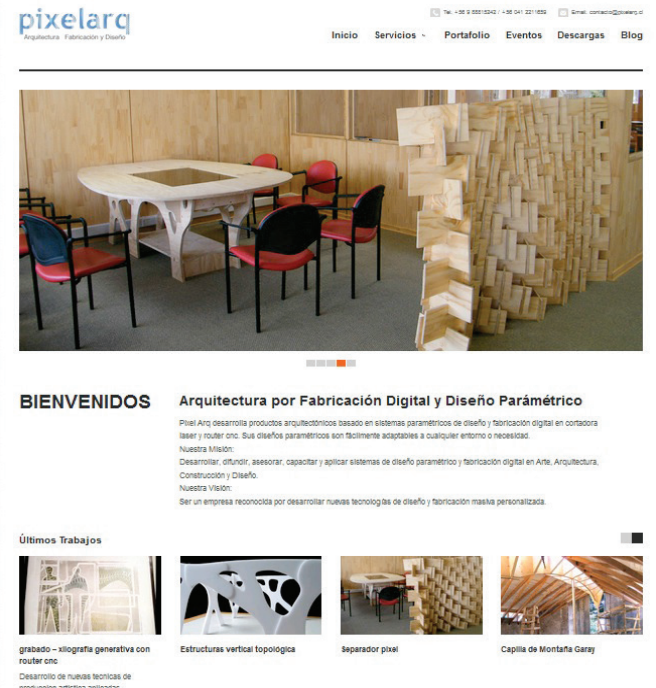

Figura 2: Web de PixelArq.

diseño parametrizable, nace Proyecto MALLA como una idea de emprendimiento impulsada por la Arquitecto Paula Ignacia Ulloa 
Aguayo, socia fundadora de Galaxia Arquitectos y el programador y estudiante de arquitectura Braulio Gatica Laurie.

MALLA es la adaptación del sistema grid-shell en módulos estandarizado plegables, originados en Europa y adaptados a la realidad constructiva del país mediante la utilización de materiales locales y el desarrollo de nuevos detalles constructivos de posición variable, diseñados especialmente para este tipo de estructuras, donde vectores desde diferentes direcciones, se articulan para generar espacialidades interesantes capaces de ser aplicadas en procesos constructivos reales. La unión de estos módulos crea una gran retícula deformable, la cual se adapta a los requerimientos espaciales del diseñador, logrando estructuras de cubiertas innovadoras con potencial de ser insertadas en el mercado actual como proyectos estandarizados destinados a programas de iniciativa pública $\mathrm{y} / \mathrm{o}$ privada.El proyecto ha recibido el apoyo de la Universidad del Bío-Bío y de FondefConicyt a trevés de su programa Fondef-Viu (120038), lo que ha permitido la construcción del primer prototipo y la generación de una idea de negocios vinculada.
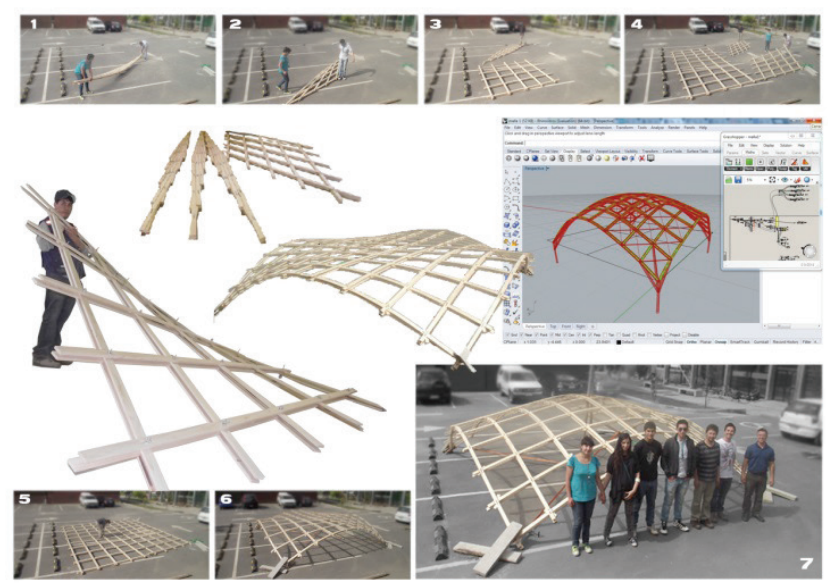

Figura 3: Malla Grid-shell

\section{Paramentos calados}

El estudio, dirigido por el egresado de arquitectura William Fuentes, consiste en experimentar un proceso de diseño y fabricación digital de paramentos calados, probando diferentes formas de adaptación y control en distintas funciones y alternativas. La experimentación en los paramentos calados pretende ser una búsqueda para lograr elementos de diseño en fachadas como celosías o en divisiones de espacios como podrían ser los biombos. Este estudio podría aportar en el control radiación solar en fachadas. Actualmente se han elaborado diversas programaciones y prototipos materiales, que se comienzan a distribuir.

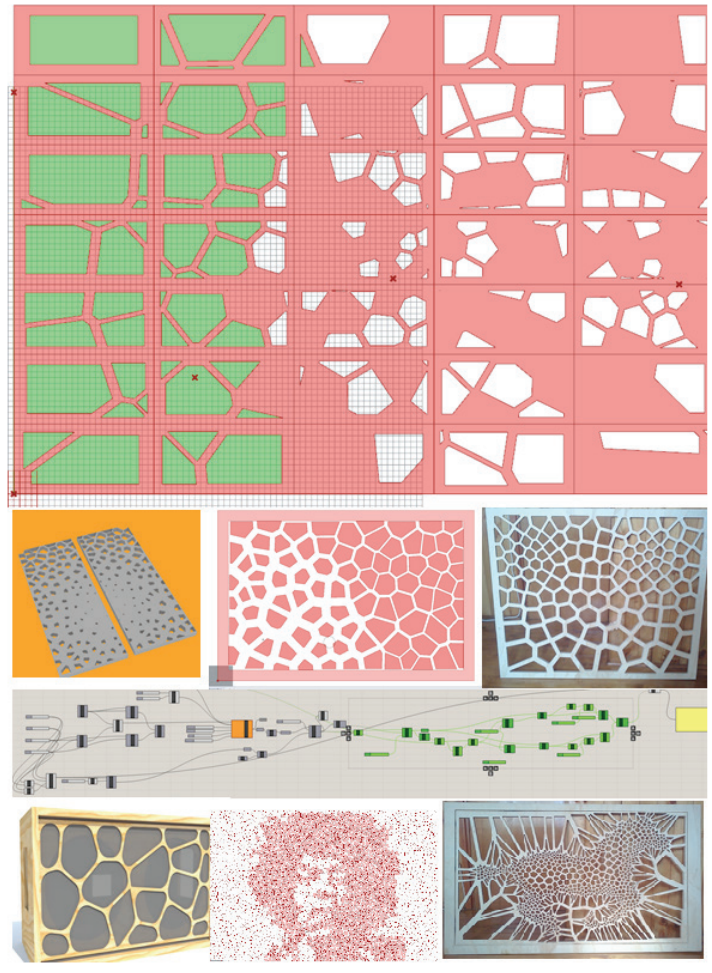

Figura 4: Paramentos Calados

\section{Plegadores de viento}

Desde la simplicidad constructiva y la autonomía estructural, nacen las directrices que rigen el proceso de desarrollo para la confección de un módulo de "Micro Espacio" que cobije a un usuario desde la perceptualidad y logre establecer un mayor grado de apropiación (un lugar) en escenarios de bastedad, como lo pudiesen ser una playa, explanadas y/o parques urbanos. Especialmente en sectores, como la costa del Centro-Sur de Chile, de amplio paisajes recreativos pero difíciles condiciones climáticas, en particular de viento, otorgando de este modo una protección temporal. Presentándose el objeto en desarrollo, como un elemento autónomo y honesto al plegarse, y erguirse como un todo formal, espacial y material. Para dialogar en una simbiosis de valoración perceptual recíproca, hacia y desde el entorno como un mobiliario urbano. Inicialmente desde una lámina de madera terciada de $11 \mathrm{~mm}$., se espera desarrollar en materiales de mayor durabilidad.

La exploración transita hacia nuevas posibilidades desde la materia hacia la forma. Incorporando en el desarrollo, algoritmos paramétricos para la generación de una patrones, que serán sustraídos al material, para una condición dúctil, y una nueva concepción geométrica. Como nuevas oportunidades de entendimiento y reflexiones materiales, en sus restricciones como en sus posibilidades donde se 

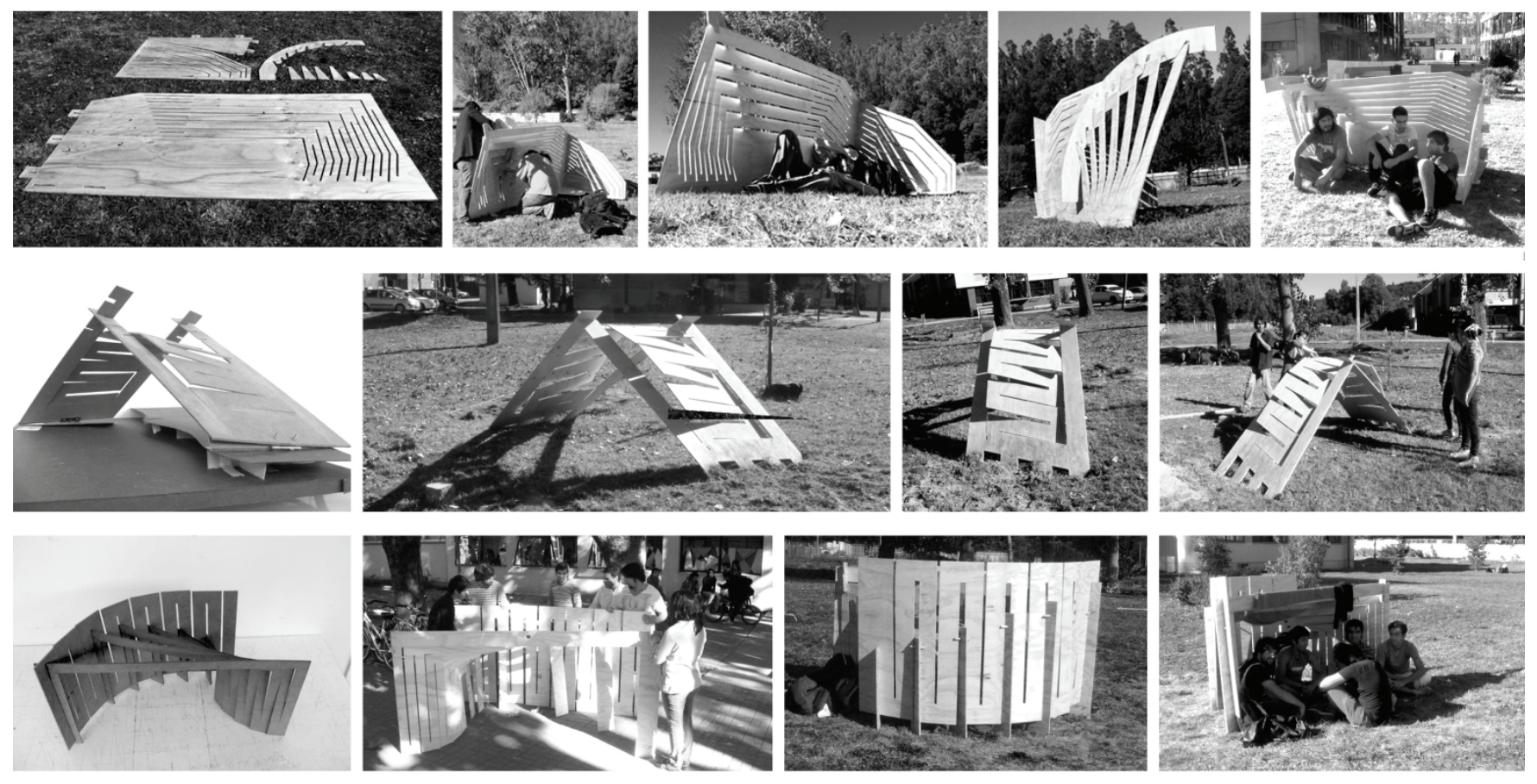

Figura 5: Plegadores de Viento.

exponen nuevas concepciones de trabajo para el ámbito arquitectónico.

La exploración se inspira primeramente en una investigación sobre el cambio de paradigma representacional que implican las composiciones plegadas y sus potencialidades pedagógicas (Chiarella, 2011) y luego de una experimentación en seminario de título en arquitectura de Alexis Salinas. Se experimentan posteriormente en un ejercicio de taller de tercer año, en el cual se desarrollan nuevas versiones de "Plegadores de Viento" (Windfolders), que pueden fabricarse en distintos materiales para transporte e instalar, o disponer transitoriamente en el lugar (Fig.4).

\section{Desarrollo}

Las experiencias descritas utilizan conjuntamente diseño paramétrico y fabricación digital. Aplicando nuevas tecnologías de proyecto y construcción para desarrollar componentes constructivos variables y masificables. Lo que implica utilización de software avanzado y nuevos equipos, pero mas que nada actitudes creativas y aproximaciones industriales para la edificación. Combinando el desarrollo de propuestas novedosas con la funcionalidad, costos y factibilidad de ejecución.

El modelo de negocio estudiado para la inserción de nuevos producto en el mercado nacional, basa sus ingresos en el diseño y la venta directa de los componentes. Para esto, el enfoque principal esta dirigido a clientes que requieran incorporar en sus proyectos componentes mas eficientes, como las cubiertas espaciales atractivas, de alto valor estético. Dentro de este mercado global, encontramos en instituciones de carácter público, especialmente municipalidades; un potencial cliente dispuesto a implementar complementos en proyectos locales. Aprovechando la agilidad en diseño que ofrece la parametrización de la estructura y la confección industrializada de sus componentes, es que se pretende trabajar en proyectos estandarizado para ofrecer a dichas instituciones, los cuales serán licitados y construidos.

Las experiencias desarrolladas se caracterizan por la exploración de una unidad constructiva variable, en algunos formando conjuntos (como las tulipas o la grid-shell) o de manera independiente (como los plegadores). Diferenciándose en la complejidad constructiva, como también en el diseño y funcionalidad. Lo que demuestra la versatilidad de las tecnologías y el impulso a la creatividad. Aunque también se verifica que las propuestas son incipientes y difícilmente comprobables por su carácter tangencial dentro de la industria de la construcción. De modo, que una alternativa por explorar es un desarrollo en sentido inverso, es decir partir de un problema existente en la edificación, y no de motivaciones individuales como ha sido hasta ahora. Seguramente esto implica desarrollos más restringidos, pero más eficaces y masificables.

\section{Conclusiones}

Estas iniciativas se caracterizan por una combinación de exploración conceptual, experimentación de tecnologías digitales avanzadas y una propuesta de masificación con la colaboración empresarial. La estrategia de diseño comienza con una conceptualización amplia y variable para acoger diversas funciones 
en situaciones distintas, sin embargo debe ser ilustrada en algunos ejemplos con modelos digitales tridimensionales para definir rangos geométricos y materiales. Posteriormente es necesario revisar posibilidades tecnológicas de programación y ejecución digital, probando algunas configuraciones simples, hasta desarrollar un sistema general. Estableciendo las variaciones principales como parámetros globales. Desarrollando luego planos de fabricación vinculados al diseño parametrico para modificar materiales y detalles directamente, asi como instrucciones de montaje para su instalación. Integrados finalmente en un proceso de ejecución y divulgación de los componentes. El repertorio de experiencias revela una aproximación de diseño basado en el conocimiento de masificación, como un ciclo de retroalimentación virtuosa, sustentado en avanzadas tecnologías digitales para proveer componentes variables al campo de la edificación

\section{Agradecimientos}

Fondecyt 1100374, 1080328, 3110025, CD-INES, DDE U.Bio-Bio, FONDEF-VIU.

\section{Referencias}

Stacey M., 2004, Digital Fabricators, Ed. University of Waterloo School of Architecture Press, Waterloo.

Woodbury R., 2010, Elements of Parametric Design, Routledge: New York.

Kieran S. and Timberlake J., 2004, Refabricating Architecture, How Manufacturing Methodologies Are Poised to Transform Building Construction, Ed. McGraw-Hill: New York.

Gramazio F. and Kohler M.; 2008, Digital Materiality in Architecture, Muller Publishers: Baden

García R. and Turkienicz B., 2010, Generative House: Exploration of Digital Fabrication and Generative System for Low - cost Housing in Southern Brazil, Proceedings of the 14th Congress SIGRADI, pp. 384387.

García R., Lagos R., Salcedo P., Ramos M., Labarca C. and Bruscato U. 2009, Precise emotions: Digital fabrication in the teaching of architecture. Arquiteturarevista v. 5, pp. 122-136

Davis D., Burry J. and Burry M., 2011, Understanding Visual Scripts: Improving collaboration through modular programming, in IJAC vol. 9 - no. 4, pp. 361-375..

Chiarella $\mathrm{M}$, Pliegues, despliegues y repliegues. Didáctica proyectual e instrumentos de Ideación" XV Congreso SIGraDi 2011 "Cultura Aumentada" FADU-UNL, 16-18/11 